\title{
Stability analysis for second-order stochastic neutral partial functional systems subject to infinite delays and impulses
}

\author{
Feng Jiang ${ }^{1 *}$, Hua Yang ${ }^{2,3}$ and Tianhai Tian ${ }^{4}$
}

\section{"Correspondence:}

fjiang@zuel.edu.cn

'School of Statistics and

Mathematics, Zhongnan University

of Economics and Law, Hubei,

430073, China

Full list of author information is

available at the end of the article

\begin{abstract}
The paper is concerned with stability for second-order stochastic neutral partial functional systems subject to infinite delays and impulses. Some sufficient conditions ensuring $p$ th moment exponential stability of the second-order stochastic systems are given by gaining a new integral inequality with impulses. Some earlier results are generalized and improved.
\end{abstract}

\section{Introduction}

The role played by second-order system in the dynamics of physical and biological systems has been an issue of growing interest and discussion. Recent research has also focused on the relative dominance of existence, uniqueness, and stability behavior of second-order systems [1-5].

In the real world, noise plays an important role in comprehending phenomena that can be very hard to describe by deterministic systems. The dynamical behavior of stochastic partial differential systems (SPDSs) has been discussed [6-11]. Sakthivel et al. [12, 13] studied pseudo almost automorphic mild solutions to stochastic fractional equations by a fixed point strategy and discussed complete controllability of stochastic evolution equations with jumps, respectively. Recently, $[14,15]$ discussed the existence, uniqueness, and stability of mild solutions to stochastic neutral partial functional systems (SNPFSs). Meanwhile, second-order SPDSs [16] are often used to model charge on a condenser, mechanical vibrations, etc. Liang and Guo [17] discussed the asymptotic behavior for second-order stochastic evolution equations with memory. Sakthivel and Ren [18] studied exponential stability of second-order stochastic evolution equations with Poisson jumps by fixed point theory. In addition, since sudden variations often exist in practice, it is necessary to model the noise source by a sequence of impulses. For instance, the stability for first-order impulsive SPDSs was discussed by using integral inequalities in [19-21]. The authors in [22-24] discussed the existence, controllability, and stability of mild solutions of second-order impulsive stochastic systems.

The papers mentioned above focus on stochastic systems with finite delays. Recently, the study of stochastic systems with infinite delay [25-29] has also been reported. For example, Ren and Sakthivel [30] discussed existence, uniqueness, and stability of a mild solution for second-order neutral stochastic evolution systems with infinite delay and Poisson

(c) 2016 Jiang et al. This article is distributed under the terms of the Creative Commons Attribution 4.0 International License (http://creativecommons.org/licenses/by/4.0/), which permits unrestricted use, distribution, and reproduction in any medium, provided you give appropriate credit to the original author(s) and the source, provide a link to the Creative Commons license, and indicate if changes were made. 
jumps by fixed point theory. Yue [31] discussed the existence of mild solutions of secondorder neutral impulsive stochastic evolution systems with infinite delay by contraction theory. The authors in $[32,33]$ extended second-order stochastic systems with infinite delay to second-order jump systems and second-order integro-differential systems.

With motivation from the above discussions, in order to overcome the difficulty of impulses and infinite delays, in this paper we first establish a new integral inequality with respect to impulses and infinite delays, which makes an important contribution to stability analysis of the second-order SNPFSs subject to infinite delays and impulses. The integral inequality is new and different from the above papers since the impulses and infinite delays exist in the system. The inequality with finite delays in [19-21,24] is extended to the inequality with infinite delays and impulses such that it is effective for the stochastic systems with infinite delays and impulses. By the new inequality, together with the stochastic analysis technique, some sufficient conditions for stability are obtained.

The rest of this paper is organized as follows. In Section 2, some preliminaries are introduced. Section 3 presents stability results by establishing a new integral inequality with impulses and infinite delays and stochastic analysis technique. In Section 4, we give an example to show the effectiveness of the obtained results.

\section{Preliminaries}

In this paper, $\left(\Omega, \mathfrak{F},\left\{\mathfrak{F}_{t}\right\}_{t \geq 0}, \mathcal{P}\right)$ is a complete probability space equipped with some filtration $\mathfrak{F}_{t}(t \geq 0)$ satisfying the usual conditions, i.e., the filtration is right continuous and $\mathfrak{F}_{0}$ contains all $\mathfrak{F}$-null sets. Let $\Xi_{1}$ and $\Xi_{2}$ be two real separable Hilbert spaces and $L\left(\Xi_{2}, \Xi_{1}\right)$ be the space of bounded linear operators from $\Xi_{2}$ to $\Xi_{1} \cdot\|\cdot\|$ denotes the norms in $\Xi_{1}, \Xi_{2}$, and $L\left(\Xi_{2}, \Xi_{1}\right) \cdot \mathcal{C}\left((-\infty, 0], \Xi_{1}\right)$ is the space of all bounded and continuous functions $\varphi$ from $(-\infty, 0]$ to $\Xi_{1}$ with the norm $\|\cdot\|_{\mathcal{C}}=\sup _{-\infty<\theta \leq 0}\|\varphi(\theta)\| \cdot \mathfrak{J}$ is the family of all $\mathfrak{F}_{t}(t \geq 0)$-measurable and $\mathcal{C}\left((-\infty, 0], \Xi_{1}\right)$-valued random variables. Let the stochastic process $w(t)$ be a $Q$-Wiener process and $\sigma \in L\left(\Xi_{2}, \Xi_{1}\right)$ be a Q-Hilbert-Schmidt operator with $\|\sigma\|_{L_{2}^{0}}^{2}=\operatorname{tr}\left(\sigma Q \sigma^{*}\right)<+\infty . L_{2}^{0}\left(\Xi_{2}, \Xi_{1}\right)$ denotes the space of all Q-Hilbert- Schmidt operators $\sigma: \Xi_{2} \rightarrow \Xi_{1}$. For details, we can refer to [6] and the references therein.

Consider the following second-order stochastic neutral partial functional systems (SNPFSs) subject to infinite delays and impulses:

$$
\left\{\begin{array}{l}
d\left[y^{\prime}(t)-\mu\left(t, y_{t}\right)\right]=\left[A y(t)+f\left(t, y_{t}\right)\right] d t+g\left(t, y_{t}\right) d w(t), \quad t \geq 0, t \neq t_{j}, j=1,2, \ldots \\
\Delta y\left(t_{j}\right)=I_{j}\left(y\left(t_{j}^{-}\right)\right), \quad j=1,2, \ldots \\
\Delta y^{\prime}\left(t_{j}\right)=\widetilde{I}_{j}\left(y\left(t_{j}^{-}\right)\right), \quad j=1,2, \ldots \\
y_{0}(\cdot)=\varphi \in \mathfrak{J}, \quad y^{\prime}(0)=\Phi
\end{array}\right.
$$

where $\Phi$ is also an $\mathfrak{F}_{0}$-measurable $\Xi_{1}$-valued random variable independent of the Wiener process $w(t) . A: D(A) \subset \Xi_{1} \rightarrow \Xi_{1}$ is the infinitesimal generator of a strongly continuous cosine family on $\Xi_{1} ; f, \mu:[0,+\infty) \times \mathfrak{J} \rightarrow \Xi_{1}, g:[0,+\infty) \times \mathfrak{J} \rightarrow L_{2}^{0}\left(\Xi_{2}, \Xi_{1}\right), y_{t}:(-\infty, 0] \rightarrow$ $\Xi_{1}, y_{t}(\theta)=y(t+\theta)(t \geq 0), 0<t_{1}<t_{2}<\cdots<t_{j}<\cdots$, and $\lim _{j \rightarrow+\infty} t_{j}=+\infty . I_{j}, \widetilde{I}_{j}: \mathfrak{J} \rightarrow H$, $\Delta \xi(t)=\xi\left(t^{+}\right)-\xi\left(t^{-}\right)$, where $\xi\left(t^{+}\right)$and $\xi\left(t^{-}\right)$denote the right and left limits of $\xi$ at $t$, respectively.

Let $\{C(t): t \in R\} \subset L\left(\Xi_{1}, \Xi_{1}\right)$ be a strongly continuous cosine family (see [5] and the references therein) and the corresponding strongly continuous sine family $\{S(t): t \in R\} \subset$ $L\left(\Xi_{1}, \Xi_{1}\right)$ be defined by $S(t) y=\int_{0}^{t} C(s) y d s, t \in R, y \in \Xi_{1}$. The generator $A: \Xi_{1} \rightarrow \Xi_{1}$ of $\{C(t): t \in R\}$ is defined by $A y=\left.\frac{d^{2}}{d t^{2}} C(t) y\right|_{t=0}$ for all $y \in D(A)=\left\{y \in \Xi_{1}: C(\cdot) y \in C^{2}\left(R, \Xi_{1}\right)\right\}$. 
Lemma 1 ([31]) Let $A$ be the infinitesimal generator of a cosine family of operators $\{C(t)$ : $t \in R\}$. Then:

(i) There exist $\delta^{*} \geq 1$ and $\beta \geq 0$ such that $\|C(t)\| \leq \delta^{*} e^{\beta t}$ and hence $\|S(t)\| \leq \delta^{*} e^{\beta t}$.

(ii) For $0 \leq s \leq r<+\infty, A \int_{s}^{r} S(u) y d u=[C(r)-C(u)] y$.

(iii) There exists $\delta^{* *} \geq 1$ such that for $0 \leq r \leq s<+\infty\|S(s)-S(r)\| \leq \delta^{* *}\left|\int_{r}^{s} e^{\beta|\theta|} d \theta\right|$.

Lemma 2 ([6]) For any $p \geq 1$ and for an $L_{2}^{0}\left(\Xi_{2}, \Xi_{1}\right)$-valued predictable process $y(\cdot)$ we have

$$
\sup _{s \in[0, t]} E\left\|\int_{0}^{s} y(v) d w(v)\right\|^{p} \leq c_{p}\left(\int_{0}^{t}\left(E\|y(v)\|_{L_{2}^{0}}^{p}\right)^{\frac{2}{p}} d v\right)^{p / 2}, \quad t \in[0,+\infty),
$$

where $c_{p}=(p(p-1) / 2)^{p / 2}$.

Definition 1 An $\Xi_{1}$-value stochastic process $y(t)(t \in R)$ is called a mild solution of system (1), if

(i) $y(t)$ is adapted to $\mathfrak{F}_{t}(t \geq 0)$ and has a càdlàg path on $t \geq 0$ almost surely.

(ii) For $t \in[0,+\infty)$, almost surely

$$
\begin{aligned}
y(t)= & C(t) \varphi+S(t)(\xi-\mu(0, \varphi))+\int_{0}^{t} C(t-v) \mu\left(v, y_{v}\right) d v \\
& +\int_{0}^{t} S(t-v) f\left(v, y_{v}\right) d v+\int_{0}^{t} S(t-v) g\left(v, y_{v}\right) d w(v) \\
& +\sum_{0<t_{j}<t} C\left(t-t_{j}\right) I_{j}\left(y\left(t_{j}^{-}\right)\right)+\sum_{0<t_{j}<t} S\left(t-t_{j}\right) \widetilde{I}_{j}\left(y\left(t_{j}^{-}\right)\right) .
\end{aligned}
$$

Definition 2 We say there is a mild solution of system (1), or for simplicity system (1) is said to be $p$ th $(p \geq 2)$ moment exponentially stable, if there exist constants $\gamma>0$ and $N_{1}>0$ such that

$$
E\|y(t)\|^{p} \leq N_{1} e^{-\gamma t}, \quad t \geq 0, p \geq 2,
$$

for the initial data $\varphi \in \mathfrak{J}$.

\section{Main results}

In the section, we will establish exponential stability of system (1) by some integral inequalities. Thus we need to employ the following assumptions:

$\left(\mathrm{A}_{1}\right)$ For some constants $\alpha \geq 1, a>0$, and $b>0,\|C(t)\| \leq \alpha e^{-b t}$ and $\|S(t)\| \leq \alpha e^{-a t}, t \geq 0$.

$\left(\mathrm{A}_{2}\right)$ There exist constants $L_{i}>0(i=0,1,2)$ and a function $\kappa:(-\infty, 0] \rightarrow[0,+\infty)$ with $\int_{-\infty}^{0} \kappa(t) d t=1$ and $\int_{-\infty}^{0} \kappa(t) e^{-\hbar t} d t<+\infty$ for $\hbar>0$, such that

$$
\begin{aligned}
& \left\|\mu\left(t, y_{1}\right)-\mu\left(t, y_{2}\right)\right\| \leq L_{0} \int_{-\infty}^{0} \kappa(\vartheta)\left\|y_{1}(t+\vartheta)-y_{2}(t+\vartheta)\right\| d \vartheta, \\
& \left\|f\left(t, y_{1}\right)-f\left(t, y_{2}\right)\right\| \leq L_{1} \int_{-\infty}^{0} \kappa(\vartheta)\left\|y_{1}(t+\vartheta)-y(t+\vartheta)\right\| d \vartheta, \\
& \left\|g\left(t, y_{1}\right)-g\left(t, y_{2}\right)\right\|_{\mathcal{L}_{2}^{0} \leq} \leq L_{2} \int_{-\infty}^{0} \kappa(\vartheta)\left\|y_{1}(t+\vartheta)-y_{2}(t+\vartheta)\right\| d \vartheta,
\end{aligned}
$$

and $\mu(t, 0)=f(t, 0)=g(t, 0)=0$ for any $y_{1}, y_{2} \in \mathfrak{J}$ and $t \geq 0$. 
$\left(\mathrm{A}_{3}\right)$ For $y_{1}, y_{2} \in \Xi_{1}$ and $\sum_{j=1}^{+\infty} \alpha_{j}<+\infty, \sum_{j=1}^{+\infty} \beta_{j}<+\infty$, there exist positive numbers $\alpha_{j}, \beta_{j}$ $(j=1,2, \ldots)$ such that

$$
\begin{aligned}
& \left\|I_{j}\left(y_{1}\right)-I_{j}\left(y_{2}\right)\right\| \leq \alpha_{j}\left\|y_{1}-y_{2}\right\|, \\
& \left\|\widetilde{I}_{j}\left(y_{1}\right)-\widetilde{I}_{j}\left(y_{2}\right)\right\| \leq \beta_{j}\left\|y_{1}-y_{2}\right\|,
\end{aligned}
$$

and $I_{j}(0)=\widetilde{I}_{j}(0)=0$.

Remark 1 References [8,31] discussed the existence of mild solutions of second-order stochastic neutral partial functional equations with infinite delay or with finite delay, respectively. Similarly, we can show that for system (1) there exists a unique mild solution under the conditions $\left(A_{1}\right)-\left(A_{3}\right)$. Also, it is obvious that system (1) has a unique trivial mild solution when the initial value $\varphi=0$.

To obtain exponential stability of system (1), we first establish the following integral inequality with impulses and infinite delays.

Lemma 3 Suppose that $\eta_{1}, \eta_{2} \in(0, \hbar]$ and there exist constants $\zeta_{i}>0(i=1,2,3,4)$ and a function $\Psi: R \rightarrow[0,+\infty)$ such that $\frac{\zeta_{3}}{\eta_{1}}+\frac{\zeta_{4}}{\eta_{2}}+\sum_{j=1}^{+\infty}\left(a_{j}+b_{j}\right)<1$, and

$$
\Psi(t) \leq\left\{\begin{aligned}
\zeta_{1} e^{-\eta_{1} t}+\zeta_{2} e^{-\eta_{2} t}+\zeta_{3} \int_{0}^{t} e^{-\eta_{1}(t-s)} \int_{-\infty}^{0} \kappa(\vartheta) \Psi(s+\vartheta) d \vartheta d s \\
\quad+\zeta_{4} \int_{0}^{t} e^{-\eta_{2}(t-s)} \int_{-\infty}^{0} \kappa(\vartheta) \Psi(s+\vartheta) d \vartheta d s \\
\quad+\sum_{t_{j}<t} a_{j} e^{-\eta_{1}\left(t-t_{j}\right)} \Psi\left(t_{j}^{-}\right)+\sum_{t_{j}<t} b_{j} e^{-\eta_{2}\left(t-t_{j}\right)} \Psi\left(t_{j}^{-}\right), \quad t \geq 0 \\
\zeta_{1} e^{-\eta_{1} t}+\zeta_{2} e^{-\eta_{2} t}, \quad t \in(-\infty, 0]
\end{aligned}\right.
$$

holds. Then $\Psi(t) \leq N_{2} e^{-\delta t}, t \in(-\infty,+\infty)$, where $\delta \in\left(0, \eta_{1} \wedge \eta_{2}\right)$ is a root of the integral equation: $\left(\frac{\zeta 3}{\eta_{1}-\delta}+\frac{\zeta_{4}}{\eta_{2}-\delta}\right) \int_{-\infty}^{0} \kappa(\vartheta) e^{-\delta \vartheta} d \vartheta+\sum_{j=1}^{+\infty}\left(a_{j}+b_{j}\right)=1$ and $N_{2}=\max \left\{\zeta_{1}+\zeta_{2}\right.$, $\left.\frac{\zeta_{1}\left(\eta_{1}-\delta\right)}{\zeta_{3} \int_{-\infty}^{0} \kappa(\vartheta) e^{-\delta \vartheta} d \vartheta}, \frac{\zeta_{2}\left(\eta_{2}-\delta\right)}{\zeta_{4} \int_{-\infty}^{0} \kappa(\vartheta) e^{-\delta \vartheta} d \vartheta}\right\}>0$.

Proof Let $F(\zeta)=\left(\frac{\zeta_{3}}{\eta_{1}-\zeta}+\frac{\zeta_{4}}{\eta_{2}-\zeta}\right) \int_{-\infty}^{0} \kappa(\vartheta) e^{-\zeta \vartheta} d \vartheta+\sum_{j=1}^{+\infty}\left(a_{j}+b_{j}\right)-1$, then it is obvious that there exists a positive constant $\delta \in\left(0, \eta_{1} \wedge \eta_{2}\right)$, such that $F(\delta)=0$.

For any $\varepsilon>0$ and let

$$
N_{\varepsilon}=\max \left\{\zeta_{1}+\zeta_{2}+\varepsilon, \frac{\left(\eta_{1}-\delta\right)\left(\zeta_{1}+\varepsilon\right)}{\zeta_{3} \int_{-\infty}^{0} \kappa(\vartheta) e^{-\delta \vartheta} d \vartheta}, \frac{\left(\eta_{2}-\delta\right)\left(\zeta_{2}+\varepsilon\right)}{\zeta_{4} \int_{-\infty}^{0} \kappa(\vartheta) e^{-\delta \vartheta} d \vartheta}\right\}>0 .
$$

Now we only need to claim that (3) implies

$$
\Psi(t) \leq N_{\varepsilon} e^{-\delta t}, \quad t \in(-\infty,+\infty)
$$

Obviously, for $t \in(-\infty, 0]$, (5) holds. Next we will prove (5) by the contradiction method. Assume that there exists a $t_{1}>0$ such that

$$
\Psi(t)<N_{\varepsilon} e^{-\delta t}, \quad t \in\left(-\infty, t_{1}\right), \quad \Psi\left(t_{1}\right)=N_{\varepsilon} e^{-\delta t_{1}} .
$$


Note that $\delta \in\left(0, \eta_{1} \wedge \eta_{2}\right)$, from (3) we have

$$
\begin{aligned}
\Psi\left(t_{1}\right) \leq & \zeta_{1} e^{-\eta_{1} t_{1}}+\zeta_{2} e^{-\eta_{2} t_{1}}+\zeta_{3} N_{\varepsilon} \int_{0}^{t_{1}} e^{-\eta_{1}\left(t_{1}-s\right)} \int_{-\infty}^{0} \kappa(\vartheta) e^{-\delta(s+\vartheta)} d s \\
& +\zeta_{4} N_{\varepsilon} \int_{0}^{t_{1}} e^{-\eta_{2}\left(t_{1}-s\right)} \int_{-\infty}^{0} \kappa(\vartheta) e^{-\delta(s+\vartheta)} d \vartheta d s \\
& +N_{\varepsilon} \sum_{t_{j}<t} a_{j} e^{-\eta_{1}\left(t_{1}-t_{j}\right)} e^{-\delta t_{j}}+N_{\varepsilon} \sum_{t_{j}<t} b_{j} e^{-\eta_{2}\left(t_{1}-t_{j}\right)} e^{-\delta t_{j}} \\
\leq & \left(\zeta_{1}-\frac{N_{\varepsilon} \zeta_{3}}{\eta_{1}-\delta} \int_{-\infty}^{0} \kappa(\vartheta) e^{-\delta \vartheta} d \vartheta\right) e^{-\eta_{1} t_{1}}+\left(\zeta_{2}-\frac{N_{\varepsilon} \zeta_{4}}{\eta_{2}-\delta} \int_{-\infty}^{0} \kappa(\vartheta) e^{-\delta \vartheta} d \vartheta\right) e^{-\eta_{2} t_{1}} \\
& +\left(\frac{\zeta_{3}}{\eta_{1}-\delta} \int_{-\infty}^{0} \kappa(\vartheta) e^{-\delta \vartheta} d \vartheta+\frac{\zeta_{4}}{\eta_{2}-\delta} \int_{-\infty}^{0} \kappa(\vartheta) e^{-\delta \vartheta} d \vartheta\right. \\
& +\sum_{j=1}^{+\infty}\left(a_{j}+b_{j}\right) N_{\varepsilon} e^{-\delta t_{1}} .
\end{aligned}
$$

By (4), we have

$$
\zeta_{1}-\frac{\zeta_{3} N_{\varepsilon}}{\eta_{1}-\delta} \int_{-\infty}^{0} \kappa(\vartheta) e^{-\delta \vartheta} d \vartheta \leq \zeta_{1}-\frac{\zeta_{3}}{\eta_{1}-\delta} \int_{-\infty}^{0} \kappa(\vartheta) e^{-\delta \vartheta} d \vartheta \frac{\left(\eta_{1}-\delta\right)\left(\zeta_{1}+\varepsilon\right)}{\zeta_{3} \int_{-\infty}^{0} \kappa(\vartheta) e^{-\delta \vartheta} d \vartheta}<0
$$

and

$$
\zeta_{2}-\frac{N_{\varepsilon} \zeta_{4}}{\eta_{2}-\delta} \int_{-\infty}^{0} \kappa(\vartheta) e^{-\delta \vartheta} d \vartheta \leq \zeta_{2}-\frac{\zeta_{4}}{\eta_{2}-\delta} \int_{-\infty}^{0} \kappa(\vartheta) e^{-\delta \vartheta} d \vartheta \frac{\left(\eta_{2}-\delta\right)\left(\zeta_{2}+\varepsilon\right)}{\zeta_{4} \int_{-\infty}^{0} \kappa(\vartheta) e^{-\delta \vartheta} d \vartheta}<0
$$

Thus, (7) yields $\Psi\left(t_{1}\right)<N_{\varepsilon} e^{-\delta t_{1}}$, which contradicts (6), that is, (5) holds. Since $\varepsilon>0$ is small enough, by (5), we have $\Psi(t) \leq N_{2} e^{-\delta t}, t \geq 0$, where $N_{2}=\max \left\{\zeta_{1}+\zeta_{2}, \frac{\zeta_{1}\left(\eta_{1}-\delta\right)}{\zeta_{3} \int_{-\infty}^{0} \kappa(\vartheta) e^{-\delta \vartheta} d \vartheta}\right.$, $\left.\frac{\zeta_{2}\left(\eta_{2}-\delta\right)}{\zeta_{4} \int_{-\infty}^{0} \kappa(\vartheta) e^{-\delta \vartheta} d \vartheta}\right\}>0$. The proof is completed.

Remark 2 Lemma 1, which is different from the lemmas of [21, 24, 29], plays an important role here. The main lemmas in $[21,24,29]$ cannot be applied in the present study because of the effects of impulses and infinite delays. The main lemma of [21] aims at first-order stochastic systems, while that of [24] focuses on finite constant delay. Our lemma is effective to establish the stability of system (1). It is clear that the lemma can deal with impulses and infinite delay terms.

Theorem 1 Assume that $\left(\mathrm{A}_{1}\right)-\left(\mathrm{A}_{3}\right)$ hold and $a, b \in(0, \hbar]$, and for $p \geq 2$,

$$
\begin{aligned}
& 7^{p-1} \alpha^{p} L_{0}^{p} b^{-p}+7^{p-1} \alpha^{p} L_{1}^{p} a^{-p}+7^{p-1} \alpha^{p} L_{2}^{p} a^{-\frac{p}{2}}\left(\frac{p(p-1)}{2}\right)^{\frac{p}{2}}\left(\frac{2(p-1)}{p-2}\right)^{1-\frac{p}{2}} \\
& +7^{p-1} \alpha^{p}\left(\sum_{j=1}^{+\infty} \beta_{j}\right)^{p}+7^{p-1} \alpha^{p}\left(\sum_{j=1}^{+\infty} \alpha_{j}\right)^{p}<1 .
\end{aligned}
$$

Then system (1) is pth moment exponentially stable. Especially, when $p=2$ system (1) is mean square exponentially stable provided $\alpha^{2} L_{0}^{2} b^{-2}+\alpha^{2} L_{1}^{2} a^{-2}+\alpha^{2} L_{2}^{2} a^{-1}+\alpha^{2}\left(\sum_{j=1}^{+\infty} \beta_{j}\right)^{2}+$ $\alpha^{2}\left(\sum_{j=1}^{+\infty} \alpha_{j}\right)^{2}<1 / 7$. 
Proof By (2), we have

$$
\begin{aligned}
E\|y(t)\|^{p}= & E \| C(t) \varphi+S(t)(\Phi-\mu(0, \varphi))+\int_{0}^{t} C(t-\varpi) \mu\left(\varpi, y_{\varpi}\right) d \varpi \\
& +\int_{0}^{t} S(t-\varpi) f\left(\varpi, y_{\varpi}\right) d \varpi+\int_{0}^{t} S(t-\varpi) g\left(\varpi, y_{\varpi}\right) d w(\varpi) \\
& +\sum_{0<t_{j}<t} C\left(t-t_{j}\right) I_{j}\left(y\left(t_{j}^{-}\right)\right)+\sum_{0<t_{j}<t} S\left(t-t_{j}\right) \widetilde{I}_{j}\left(y\left(t_{j}^{-}\right)\right) \|^{p} \\
\leq & 7^{p-1} \alpha^{p} E\|\varphi\|^{p} e^{-b t}+7^{p-1} \alpha^{p} E\|\Phi-\mu(0, \varphi)\|^{p} e^{-a t} \\
& +7^{p-1} E\left\|\int_{0}^{t} C(t-\varpi) \mu\left(\varpi, y_{\varpi}\right) d \varpi\right\|^{p} \\
& +7^{p-1} E\left\|\int_{0}^{t} S(t-\varpi) f\left(\varpi, y_{\varpi}\right) d \varpi\right\|^{p} \\
& +7^{p-1}\left\|\int_{0}^{t} S(t-\varpi) g\left(\varpi, y_{\varpi}\right) d w(\varpi)\right\|^{p} \\
& +7^{p-1} E\left\|\sum_{0<t_{j}<t} C\left(t-t_{j}\right) I_{j}\left(y\left(t_{j}^{-}\right)\right)\right\|^{p}+7^{p-1} E\left\|\sum_{0<t_{j}<t} S\left(t-t_{j}\right) \widetilde{I}_{j}\left(y\left(t_{j}^{-}\right)\right)\right\|^{p} .
\end{aligned}
$$

By $\left(A_{1}\right),\left(A_{2}\right),\left(A_{3}\right)$, and the Hölder inequality, we have

$$
\begin{aligned}
E \| & \int_{0}^{t} C(t-\varpi) \mu\left(\varpi, y_{\varpi}\right) d \varpi \|^{p} \\
& \leq \alpha^{p}\left(\int_{0}^{t}\left(e^{-b(t-\varpi)(p+1) / p}\right)^{\frac{p}{p-1}} d \varpi\right)^{p-1} \int_{0}^{t} e^{-b(t-\varpi)} E\left\|\mu\left(\varpi, y_{\varpi}\right)\right\|^{p} d \varpi \\
& \leq \alpha^{p} b^{1-p} \int_{0}^{t} e^{-b(t-\varpi)} E\left\|\mu\left(\varpi, y_{\varpi}\right)-\mu(\varpi, 0)+\mu(\varpi, 0)\right\|^{p} d \varpi \\
& \leq \alpha^{p} L_{0}^{p} b^{1-p} \int_{0}^{t} e^{-b(t-\varpi)} E\left(\int_{-\infty}^{0} \kappa(\vartheta)\|y(\varpi+\vartheta)\| d \vartheta\right)^{p} d \varpi .
\end{aligned}
$$

Similarly, we have

$$
\begin{aligned}
& E\left\|\int_{0}^{t} S(t-\varpi) f\left(\varpi, y_{\varpi}\right) d \varpi\right\|^{p} \\
& \quad \leq \alpha^{p} L_{1}^{p} a^{1-p} \int_{0}^{t} e^{-a(t-\varpi)} E\left(\int_{-\infty}^{0} \kappa(\vartheta)\|y(\varpi+\vartheta)\| d \vartheta\right)^{p} d \varpi .
\end{aligned}
$$

By $\left(A_{1}\right)$ and $\left(A_{3}\right)$, we have

$$
\begin{aligned}
\left\|\sum_{0<t_{j}<t} C\left(t-t_{j}\right) I_{j}\left(y\left(t_{j}^{-}\right)\right)\right\|^{p} & \leq \alpha^{p} E\left(\sum_{0<t_{j}<t} e^{-b\left(t-t_{j}\right)} \alpha_{j}\left\|y\left(t_{j}^{-}\right)\right\|\right)^{p} \\
& \leq \alpha^{p} E\left(\sum_{j=1}^{+\infty} \alpha_{j}^{\frac{p-1}{p}} \alpha_{j}^{\frac{1}{p}} e^{-b\left(t-t_{j}\right)}\left\|y\left(t_{j}^{-}\right)\right\|\right)^{p}
\end{aligned}
$$




$$
\begin{aligned}
& \leq \alpha^{p}\left(\sum_{j=1}^{+\infty} \alpha_{j}\right)^{p-1} \sum_{0<t_{j}<t} q_{j} e^{-p b\left(t-t_{j}\right)} E\left\|y\left(t_{j}^{-}\right)\right\|^{p} \\
& \leq \alpha^{p}\left(\sum_{j=1}^{+\infty} \alpha_{j}\right)^{p-1} \sum_{0<t_{j}<t} \alpha_{j} e^{-b\left(t-t_{j}\right)} E\left\|y\left(t_{j}^{-}\right)\right\|^{p}
\end{aligned}
$$

and

$$
\left\|\sum_{0<t_{j}<t} S\left(t-t_{j}\right) \widetilde{I}_{j}\left(y\left(t_{j}^{-}\right)\right)\right\|^{p} \leq \alpha^{p}\left(\sum_{j=1}^{+\infty} \beta_{j}\right)^{p-1} \sum_{0<t_{j}<t} \beta_{j} e^{-a\left(t-t_{j}\right)} E\left\|y\left(t_{j}^{-}\right)\right\|^{p} .
$$

From Lemma 2, we obtain

$$
\begin{aligned}
& E\left\|\int_{0}^{t} S(t-\varpi) g\left(\varpi, y_{\varpi}\right) d w(\varpi)\right\|^{p} \\
& \quad \leq c_{p} \alpha^{p}\left(\int_{0}^{t}\left(e^{-a p(t-\varpi)} E\left\|g\left(\varpi, y_{\varpi}\right)\right\|_{L_{2}^{0}}^{p}\right)^{\frac{2}{p}} d \varpi\right)^{\frac{p}{2}} \\
& \quad=c_{p} \alpha^{p}\left(\int_{0}^{t} e^{-2 a(t-\varpi)}\left(E\left\|g\left(\varpi, y_{\varpi}\right)\right\|_{L_{2}^{0}}^{p}\right)^{\frac{2}{p}} d \varpi\right)^{\frac{p}{2}} \\
& \quad \leq c_{p} \alpha^{p}\left(\int_{0}^{t} e^{-\frac{2 a(p-1)}{p-2}(t-\varpi)} d \varpi\right)^{\frac{p}{2}-1} \int_{0}^{t} e^{-a(t-\varpi)} E\left\|g\left(\varpi, y_{\varpi}\right)\right\|_{L_{2}^{0}}^{p} d \varpi \\
& \quad \leq c_{p} \alpha^{p} L_{2}^{p}\left(\frac{2 a(p-1)}{p-2}\right)^{1-\frac{p}{2}} \int_{0}^{t} e^{-a(t-\varpi)} E\left(\int_{-\infty}^{0} \kappa(\vartheta)\|y(\varpi+\vartheta)\| d \vartheta\right)^{p} d \varpi .
\end{aligned}
$$

These together with (9) yield

$$
\begin{aligned}
E\|y(t)\|^{p} & \\
\leq & 7^{p-1} \alpha^{p} E\|\varphi\|^{p} e^{-b t}+7^{p-1} \alpha^{p} E\|\Phi-\mu(0, \varphi)\|^{p} e^{-a t} \\
& +7^{p-1} \alpha^{p} L_{0}^{p} b^{1-p} \int_{0}^{t} e^{-b(t-\varpi)} \int_{-\infty}^{0} \kappa(\vartheta) E\|y(\varpi+\vartheta)\|^{p} d \vartheta d \varpi \\
& +7^{p-1} \alpha^{p} L_{1}^{p} a^{1-p} \int_{0}^{t} e^{-a(t-\varpi)} \int_{-\infty}^{0} \kappa(\vartheta) E\|y(\varpi+\vartheta)\|^{p} d \vartheta d \varpi \\
& +7^{p-1} c_{p} \alpha^{p} L_{2}^{p}\left(\frac{2 a(p-1)}{p-2}\right)^{1-\frac{p}{2}} \int_{0}^{t} e^{-a(t-\varpi)} \int_{-\infty}^{0} \kappa(\vartheta) E\|y(\varpi+\vartheta)\|^{p} d \vartheta d \varpi \\
& +7^{p-1} \alpha^{p}\left(\sum_{j=1}^{+\infty} \alpha_{j}\right)^{p-1} \sum_{0<t_{j}<t} \alpha_{j} e^{-b\left(t-t_{j}\right)} E\left\|y\left(t_{j}^{-}\right)\right\|^{p} \\
& +7^{p-1} \alpha^{p}\left(\sum_{j=1}^{+\infty} \beta_{j}\right)^{p-1} \sum_{0<t_{j}<t} \beta_{j} e^{-a\left(t-t_{j}\right)} E\left\|y\left(t_{j}^{-}\right)\right\|^{p} .
\end{aligned}
$$

Obviously, from (10) there exist two positive numbers $\alpha^{\prime}$ and $\alpha^{\prime \prime}$ such that $E\|y(t)\|^{2} \leq$ $\alpha^{\prime} e^{-b t}+\alpha^{\prime \prime} e^{-a t}$, for any $t \in(-\infty, 0]$.

Let $\breve{\zeta}_{1}=7^{p-1} \alpha^{p} E\|\varphi\|^{p}, \breve{\zeta}_{2}=7^{p-1} \alpha^{p} E\|\Phi-\mu(0, \varphi)\|^{p}, \breve{\zeta}_{3}=7^{p-1} \alpha^{p} L_{0}^{p} b^{1-p}, \breve{\zeta}_{4}=7^{p-1} \alpha^{p} L_{1}^{p} a^{1-p}+$ $7^{p-1} c_{p} \alpha^{p} L_{2}^{p}\left(\frac{2 a(p-1)}{p-2}\right)^{1-\frac{p}{2}}, \check{\eta}_{j}=7^{p-1} \alpha^{p}\left(\sum_{j=1}^{+\infty} \alpha_{j}\right)^{p-1} \alpha_{j}, \breve{\eta}_{j}=7^{p-1} \alpha^{p}\left(\sum_{j=1}^{+\infty} \beta_{j}\right)^{p-1} \beta_{j}$, If $\frac{\breve{\zeta_{3}}}{b}+\frac{\breve{\zeta_{4}}}{a}+$ 
$\sum_{j=1}^{+\infty} \breve{\eta}+\sum_{j=1}^{+\infty} \check{\eta}<1$, i.e. (8) holds, then by Lemma 3, we obtain $E\|y(t)\|^{2} \leq \hat{M} e^{-\hat{\delta} t}, t \in$ $[0,+\infty)$, where $\hat{\delta} \in\left(0, \eta_{1} \wedge \eta_{2}\right)$ and $\hat{\delta}$ is a positive root of the equation $\left(\frac{\breve{\zeta}_{3}}{b-\delta}+\frac{\breve{\zeta}_{4}}{a-\delta}\right) \int_{-\infty}^{0} \kappa(\vartheta) \times$ $e^{-\delta \vartheta} d \vartheta+\sum_{j=1}^{+\infty}\left(\alpha_{j}+\beta_{j}\right)=1, \hat{M}=\left\{7^{p-1} \alpha^{p}\left(E\|\varphi\|^{p}+E\|\Phi-\mu(0, \varphi)\|^{p}\right), 7^{p-1} \alpha^{p} b^{-p} L_{0}^{p}\right.$, $\left.7^{p-1} \alpha^{p}\left(a^{-p} L_{1}^{p}+c_{p} L_{2}^{p} a^{-\frac{p}{2}}\left(\frac{2(p-1)}{p-2}\right)^{1-\frac{p}{2}}\right)\right\}>0$. The proof is completed.

If $D\left(t, y_{t}\right) \equiv 0$, system (1) becomes the following second-order SPFDSs subject to infinite delays and being impulsive:

$$
\left\{\begin{array}{l}
d y^{\prime}(t)=\left[A y(t)+f\left(t, y_{t}\right)\right] d t+g\left(t, y_{t}\right) d w(t), \quad t \geq 0, t \neq t_{j}, j=1,2, \ldots \\
\Delta y\left(t_{j}\right)=I_{j}\left(y\left(t_{j}^{-}\right)\right), \quad \Delta y^{\prime}\left(t_{j}\right)=\widetilde{I}_{j}\left(y\left(t_{j}^{-}\right)\right), \quad j=1,2, \ldots \\
y_{0}(\cdot)=\varphi \in \mathfrak{J}, \quad y^{\prime}(0)=\Phi
\end{array}\right.
$$

Corollary 1 Under $\left(\mathrm{A}_{1}\right)-\left(\mathrm{A}_{3}\right)$, and $a, b \in(0, \hbar]$, system (11) is pth $(p \geq 2)$ moment exponentially stable provided

$$
\begin{aligned}
& 6^{p-1} \alpha^{p} L_{1}^{p} a^{-p}+6^{p-1} \alpha^{p} L_{2}^{p} a^{-\frac{p}{2}}\left(\frac{p(p-1)}{2}\right)^{\frac{p}{2}}\left(\frac{2(p-1)}{p-2}\right)^{1-\frac{p}{2}} \\
& +6^{p-1} \alpha^{p}\left(\sum_{j=1}^{+\infty} \beta_{j}\right)^{p}+6^{p-1} \alpha^{p}\left(\sum_{j=1}^{+\infty} \alpha_{j}\right)^{p}<1 .
\end{aligned}
$$

Moreover, if $p=2$ system (1) is mean square exponentially stable provided $6 \alpha^{2} L_{1}^{2} a^{-2}+$ $6 \alpha^{2} L_{2}^{2} a^{-1}+6 \alpha^{2}\left(\sum_{j=1}^{+\infty} \beta_{j}\right)^{2}+6 \alpha^{2}\left(\sum_{j=1}^{+\infty} \alpha_{j}\right)^{2}<1$.

If system (1) is not subject to impulses, system (1) becomes the following second-order SNPFSs:

$$
\left\{\begin{array}{l}
d\left[y^{\prime}(t)-\mu\left(t, y_{t}\right)\right]=\left[A y(t)+f\left(t, y_{t}\right)\right] d t+g\left(t, y_{t}\right) d w(t), \quad t \geq 0, t \neq t_{j}, j=1,2, \ldots \\
y_{0}(\cdot)=\varphi \in \mathfrak{J}, \quad y^{\prime}(0)=\Phi
\end{array}\right.
$$

Corollary 2 Under $\left(\mathrm{A}_{1}\right)-\left(\mathrm{A}_{2}\right)$, and $a, b \in(0, \hbar]$, system (13) is pth $(p \geq 2)$ moment exponentially stable provided

$$
5^{p-1} \alpha^{p} L_{0}^{p} b^{-p}+5^{p-1} \alpha^{p} L_{1}^{p} a^{-p}+5^{p-1} \alpha^{p} L_{2}^{p} a^{-\frac{p}{2}}\left(\frac{p(p-1)}{2}\right)^{\frac{p}{2}}\left(\frac{2(p-1)}{p-2}\right)^{1-\frac{p}{2}}<1 .
$$

Moreover, if $p=2$ system (1) is mean square exponentially stable provided $5 \alpha^{2} L_{0}^{2} b^{-2}+$ $5 \alpha^{2} L_{1}^{2} a^{-2}+5 \alpha^{2} L_{2}^{2} a^{-1}<1$.

Remark 3 System (1) with finite delays or without impulses has been discussed in [8, 22] and [29], respectively. In this paper, we discuss the second-order stochastic neutral partial functional differential equations subject to infinite delays and impulses, which is more general. In addition, the method of establishing stability in $[9,18,28,30,31]$ is fixed point theory. However, the method different from the above papers is to establish a new integral inequality with respect to impulses and infinite delays. Although [10, 19-21, 24, 29] also utilize the integral inequalities techniques to study stochastic partial differential systems, these inequalities cannot be applied in this paper. In the sense, Theorem 1 is a 
generalization and development of these existing results. Finally, it should be pointed out that the results are valid for system (1) with finite delays. Our results can easily be extended to second-order stochastic systems driven by a Lévy process.

Remark 4 If $4^{p-1} \alpha^{p} L_{1}^{p} a^{-p}+4^{p-1} \alpha^{p} L_{2}^{p} a^{-\frac{p}{2}}\left(\frac{p(p-1)}{2}\right)^{\frac{p}{2}}\left(\frac{2(p-1)}{p-2}\right)^{1-\frac{p}{2}}<1$, system (13) without neutral term is also $p$ th moment exponentially stable. If $\alpha^{2} L_{0}^{2} b^{-2}+5 \alpha^{2} L_{1}^{2} a^{-2}+\alpha^{2} L_{2}^{2} a^{-1}<1 / 4$, system (13) without neutral term is mean square exponentially stable.

\section{An example}

In this section, we will discuss an example to show the theoretical results. Consider the following second-order stochastic neutral partial functional systems subject to infinite delays and impulses:

$$
\begin{aligned}
& d\left[\frac{\partial}{\partial t} y(t, x)-\mu\left(t, y_{t}\right)\right]=\left[\frac{\partial^{2}}{\partial x^{2}} y(t, x)+f\left(t, y_{t}\right)\right] d t+g\left(t, y_{t}\right) d w(t), \\
& \quad t \geq 0, x \in[0, \pi], \\
& \Delta y\left(t_{j}\right)=\frac{u_{3}}{j^{2}} y\left(t_{j}^{-}\right), \quad j=1,2, \ldots, \\
& \Delta y^{\prime}\left(t_{j}\right)=\frac{u_{4}}{j^{2}} y\left(t_{j}^{-}\right), \quad j=1,2, \ldots,
\end{aligned}
$$

with $y(s)=\varphi(s) \in \mathfrak{J}, y^{\prime}(0)=\xi, y(t, 0)=y(t, \pi)=0, t \geq 0, u_{i}>0, i=0,1,2$, and $u_{3} \geq 0, u_{4} \geq 0$.

Let $\Xi_{1}=\mathfrak{L}^{2}[0, \pi]$ and $\Xi_{2}=R^{1}$ with the norm $\|\cdot\|$. Let $A=\frac{\partial^{2}}{\partial x^{2}}: \Xi_{1} \rightarrow \Xi_{1}$ with $\mathfrak{D}(A)=$ $\left\{y \in \Xi_{1}: y, \frac{\partial}{\partial x} y\right.$ be absolutely continuous, $\left.\frac{\partial^{2}}{\partial x^{2}} y \in \Xi_{1}, y(0)=y(\pi)=0\right\}$. Thus it is clear that $\|C(t)\| \leq e^{-\pi^{2} t}$ and $\|S(t)\| \leq e^{-\pi^{2} t}, t \geq 0$.

Now let

$$
\begin{aligned}
& \mu\left(t, y_{t}\right) \triangleq \frac{u_{0} \pi^{2}}{7} \int_{-\infty}^{0}(-\varpi)^{-\frac{1}{2}} e^{\pi^{2} \varpi} y(t+\varpi, x) d \varpi, \\
& f\left(t, y_{t}\right) \triangleq \frac{u_{1} \pi^{2}}{7} \int_{-\infty}^{0}(-\varpi)^{-\frac{1}{2}} e^{\pi^{2} \varpi} y(t+\varpi, x) d \varpi, \\
& g\left(t, y_{t}\right) \triangleq \frac{u_{2} \pi}{7} \int_{-\infty}^{0}(-\varpi)^{-\frac{1}{2}} e^{\pi^{2} \varpi} y((t+\varpi), x) d \varpi,
\end{aligned}
$$

for any $y_{t} \in \mathfrak{J}$. It is clear that the conditions of Theorem 1 hold with $\alpha=1, b=a=\pi^{2}, L_{0}=$ $\frac{u_{0} \pi^{2}}{7}, L_{1}=\frac{u_{1} \pi^{2}}{7}, L_{2}=\frac{u_{2} \pi}{7}, a_{j}=\frac{u_{3}}{7 j^{2}}, \beta_{j}=\frac{u_{4}}{7 j^{2}}(j=1,2, \ldots)$. Then, by Theorem 1 , system (15) is $p$ th moment exponentially stable provided

$$
u_{0}^{p}+u_{1}^{p}+u_{2}^{p}\left(\frac{p(p-1)}{2}\right)^{\frac{p}{2}}\left(\frac{2(p-1)}{p-2}\right)^{1-\frac{p}{2}}+\left(\frac{u_{3} \pi^{2}}{6}\right)^{p}+\left(\frac{u_{4} \pi^{2}}{6}\right)^{p}<7 .
$$

Especially, when $p=2$, system (15) is mean square exponentially stable provided $u_{0}^{2}+u_{1}^{2}+$ $u_{2}^{2}+\frac{u_{3}^{2} \pi^{4}}{36}+\frac{u_{4}^{2} \pi^{4}}{36}<7$.

If system (15) has no impulse term, i.e. $u_{3}=u_{4}=0$, then system (15) becomes one of the second-order neutral stochastic systems with infinite delays. Now let $\alpha=1, b=a=$ $\pi^{2}, L_{0}=\frac{u_{0} \pi^{2}}{5}, L_{1}=\frac{u_{1} \pi^{2}}{5}, L_{2}=\frac{u_{2} \pi}{5}$. Then, by Corollary 2 , system (15) without impulses is 
$p$ th moment exponentially stable provided

$$
u_{0}^{p}+u_{1}^{p}+u_{2}^{p}\left(\frac{p(p-1)}{2}\right)^{\frac{p}{2}}\left(\frac{2(p-1)}{p-2}\right)^{1-\frac{p}{2}}<5
$$

and if $u_{0}^{2}+u_{1}^{2}+u_{2}^{2}<5$, system (15) without impulses is mean square exponentially stable.

Remark 5 It is clear that the algebraic conditions of stability in the example are easily computed. The example also shows that the results of this paper are effective.

\section{Conclusions}

In this paper we have studied second-order stochastic neutral partial functional systems subject to infinite delays and impulses. We first established a new integral inequality with respect to impulses and then give some algebraic criteria to ensure $p$ th moment exponential stability of the second-order stochastic impulsive systems. The results show some earlier results are generalized and improved. Finally, an example was given to illustrate the effectiveness of the theoretical results. Further research will be to study controller design and noise stabilization as well as its possible extension to second-order stochastic systems driven by Lévy jumps.

\section{Competing interests}

The authors declare that they have no competing interests.

\section{Authors' contributions}

FJ and HY jointly carried out the study of stability of this paper and drafted the manuscript. TT provided some constructive suggestions for the improvement of this paper. All authors read and approved the final manuscript.

\footnotetext{
Author details

'School of Statistics and Mathematics, Zhongnan University of Economics and Law, Hubei, 430073, China. ${ }^{2}$ School of Mathematics \& Computer Science, Wuhan Polytechnic University, Hubei, 430023, China. ${ }^{3}$ School of Automation, Huazhong University of Science and Technology, Hubei, 430074, China. ${ }^{4}$ School of Mathematical Sciences, Monash University, Melbourne, VIC 3800, Australia.
}

\section{Acknowledgements}

The work is supported by the National Natural Science Foundation of China under Grant No. 61304067 and 11571368, the Natural Science Foundation of Hubei Province of China under Grant No. 2013CFB443, the Science and Technology Research Project of Hubei Provincial Department of Education under Grant No. Q20151705 and the Australian Research Council Future Fellowship under Grant No. FT100100748.

Received: 6 October 2015 Accepted: 23 August 2016 Published online: 30 August 2016

\section{References}

1. Tsai, C, Chen, H, Hsu, JRC: Second-order time-dependent mild-slope equation for wave transformation. Math. Probl. Eng. 2014, $341385(2014)$

2. Da Prato, G, Zabczyk, J: Second-Order Partial Differential Equations in Hilbert Spaces. Cambridge University Press, Cambridge (2002)

3. Feintuch, A: Asymptotic behaviour of infinite chains of coupled kinematic points: second-order equations. Math. Control Signals Syst. 26(3), 463-480 (2014)

4. Biles, D, Spraker, J: Uniqueness of solutions for second order differential equations. Monatshefte Math. 178(2), 165-169 (2015)

5. Travis, CC, Webb, GF: Cosine families and abstract nonlinear second order differential equations. Acta Math. Acad. Sci. Hung. 32, 76-96 (1978)

6. Da, PG, Zabczyk, J: Stochastic Equations in Infinite Dimensions. Cambridge University Press, Cambridge (1992)

7. Liu, L: Stability of Infinite Dimensional Stochastic Differential Equations with Applications. Chapman \& Hall/CRC, London (2006)

8. Mckibben, MA: Second-order neutral stochastic evolution equations with heredity. J. Appl. Math. Stoch. Anal. 2 177-192 (2004)

9. Luo, J: Fixed points and exponential stability of mild solutions of stochastic partial differential equation with delays. J. Math. Anal. Appl. 342, 753-760 (2008)

10. Chen, $\mathrm{H}$ : Integral inequality and exponential stability for neutral stochastic partial differential equations with delays. J. Inequal. Appl. 2009, 297478 (2009) 
11. Bao, J, Hou, Z, Yuan, C: Stability in distribution of mild solutions to stochastic partial differential equations. Proc. Am. Math. Soc. 138, 2169-2180 (2010)

12. Sakthivel, R, Revathi, $P$, Marshal Anthoni, S: Existence of pseudo almost automorphic mild solutions to stochastic fractional differential equations. Nonlinear Anal., Theory Methods Appl. 75, 3339-3347 (2012)

13. Sakthivel, R, Ren, Y: Complete controllability of stochastic evolution equations with jumps. Rep. Math. Phys. 68, 163-174 (2011)

14. Jiang, J, Shen, Y: A note on the existence and uniqueness of mild solutions to neutral stochastic partial functional differential equations with non-Lipschitz coefficients. Comput. Math. Appl. 61, 1590-1594 (2011)

15. Yang, X, Zhu, Q: pth moment exponential stability of stochastic partial differential equations with Poisson jumps. Asian J. Control 16, 1482-1491 (2014)

16. Arora, U, Sukavanam, N: Approximate controllability of second order semilinear stochastic system with nonlocal conditions. Appl. Math. Comput. 258, 111-119 (2015)

17. Liang, F, Guo, Z: Asymptotic behavior for second order stochastic evolution equations with memory. J. Math. Anal. Appl. 419(2), 1333-1350 (2014)

18. Sakthivel, R, Ren, Y: Exponential stability of second-order stochastic evolution equations with Poisson jumps. Commun. Nonlinear Sci. Numer. Simul. 17, 4517-4523 (2012)

19. Chen, H: Impulsive-integral inequality and exponential stability for stochastic partial differential equations with delays. Stat. Probab. Lett. 80, 50-56 (2010)

20. Long, S, Teng, L, Xu, D: Global attracting set and stability of stochastic neutral partial functional differential equations with impulses. Stat. Probab. Lett. 82, 1699-1709 (2012)

21. Yang, $\mathrm{H}$, Jiang, F: Exponential stability of mild solutions to impulsive stochastic neutral partial differential equations with memory. Adv. Differ. Equ. 2013, 148 (2013)

22. Ren, Y, Dandan, S: Second-order neutral impulsive stochastic evolution equations with delay. J. Math. Phys. 50, 102709 (2009)

23. Arthi, G, Balachandran, K: Controllability of damped second order impulsive neutral integrodifferential systems with nonlocal conditions. J. Control Theory Appl. 11, 186-192 (2013)

24. Arthi, G, Park, JH, Jung, HY: Exponential stability of second-order neutral stochastic differential equations with impulses. Int. J. Control 88(6), 1300-1309 (2015)

25. Luo, J: Stability of stochastic partial differential equations with infinite delays. J. Comput. Appl. Math. 222, 364-371 (2008)

26. Ren, Y, Hu, L, Sakthivel, R: Controllability of impulsive neutral stochastic functional differential inclusions with infinite delay. J. Comput. Appl. Math. 235, 2603-2614 (2011)

27. Sakthivel, R, Luo, J: Asymptotic stability of impulsive stochastic partial differential equations with infinite delays. J. Math. Anal. Appl. 356, 1-6 (2009)

28. Jiang, F, Shen, Y: Stability of impulsive stochastic neutral partial differential equations with infinite delays. Asian J. Control 14, 1706-1709 (2012)

29. Chen, $H$ : The asymptotic behavior for second-order neutral stochastic partial differential equations with infinite delay. Discrete Dyn. Nat. Soc. 2011, 584510 (2011)

30. Ren, Y, Sakthivel, R: Existence, uniqueness, and stability of mild solution for second-order neutral stochastic evolution equations with infinite delay and Poisson jumps. J. Math. Phys. 53, 073517 (2012)

31. Yue, C: Second-order neutral impulsive stochastic evolution equations with infinite delay. Adv. Differ. Equ. 2014, 112 (2014)

32. Palanisamy, M, Chinnathambi, R: Approximate controllability of second-order neutral stochastic differential equations with infinite delay and Poisson jumps. J. Syst. Sci. Complex. 28(5), 1033-1048 (2015)

33. Huan, D: On the controllability of nonlocal second-order impulsive neutral stochastic integro-differential equations with infinite delay. Asian J. Control 17(4), 1233-1242 (2015)

\section{Submit your manuscript to a SpringerOpen ${ }^{\circ}$ journal and benefit from:}

- Convenient online submission

Rigorous peer review

- Immediate publication on acceptance

- Open access: articles freely available online

- High visibility within the field

- Retaining the copyright to your article 